\title{
La Société de Géographie de l'Est. Une géographie militante et conviviale à la Belle Époque
}

The Geographical Society of the East. A militant and convivial Geography from «La Belle Epoque»

Die Ost Geographie Gesellschaft. Eine dynamische und gastfreundliche

Geographie der « Belle Epoque "

\section{Jean-Claude Bonnefont}

\section{(Q) OpenEdition}

\section{Journals}

Édition électronique

URL : http://journals.openedition.org/rge/4551

DOI : $10.4000 /$ rge.4551

ISSN : 2108-6478

\section{Éditeur}

Association des géographes de l'Est

Édition imprimée

Date de publication : 1 janvier 1999

ISSN : 0035-3213

\section{Référence électronique}

Jean-Claude Bonnefont, "La Société de Géographie de l'Est. Une géographie militante et conviviale à la Belle Époque », Revue Géographique de l'Est [En ligne], vol. 39 / 1 | 1999, mis en ligne le 08 septembre 2013, consulté le 08 septembre 2020. URL : http://journals.openedition.org/rge/4551 ; DOI : https:// doi.org/10.4000/rge.4551

Ce document a été généré automatiquement le 8 septembre 2020

Tous droits réservés 


\section{La Société de Géographie de l'Est. Une géographie militante et conviviale à la Belle Époque}

The Geographical Society of the East. A militant and convivial Geography from

"La Belle Epoque»

Die Ost Geographie Gesellschaft. Eine dynamische und gastfreundliche

Geographie der «Belle Epoque "

Jean-Claude Bonnefont

1 Nancy a participé, avec un certain succès, au grand mouvement qui a donné naissance, dans le dernier quart du XIXe siècle, à la floraison des sociétés de géographie en France. En marge de la géographie universitaire officielle, mais sans se couper d'elle, la Société de Géographie de Nancy a joué un rôle fédérateur important pour tous les courants très divers qui contribuaient alors au progrès des connaissances géographiques. Elle a en particulier aidé à combler le fossé qui s'était alors creusé entre la géographie humaine, enseignée dans les Facultés de Lettres, et la géographie physique, qui faisait la synthèse de diverses disciplines enseignées dans les Facultés des Sciences. N'excluant personne, elle a réussi un moment à démultiplier l'action d'une petite poignée de géographes, en leur offrant une caisse de résonance remarquable.

2 L'étude des 35 années d'existence de cette société, jusqu'à la Première Guerre mondiale, menée à travers une analyse approfondie de ses bulletins (1879-1913), nous apporte de multiples enseignements : sur le milieu régional lorrain à cette époque, sur les diverses conceptions qu'on pouvait avoir alors de la géographie, sur les hommes qui ont contribué alors à la faire avancer et sur la façon dont elle s'est peu à peu dégagée d'une vulgarisation anecdotique et journalistique, pour devenir plus scientifique. Elle n'évolue pas en vase clos : les contacts avec l'extérieur sont riches et fréquents. Mais la Lorraine est alors suffisamment entreprenante et dynamique pour qu'y apparaisse une synthèse originale, qui portera surtout ses fruits après la Première Guerre mondiale, quand les effectifs des géographes seront devenus plus nombreux. 
Nous examinerons successivement :

- la vie de la société et son insertion régionale,

- l'apport du bulletin de la société à la géographie française,

- la constitution d'une équipe soudée, aux compétences complémentaires,

• l'émergence d'une géographie consciente d'elle même.

\section{La vie de la société et son insertion régionale (fig. 1)}

La Société de Géographie de l'Est a été créée au début de l'année 1879, dans des conditions qui sont relatées dans le bulletin de 1879, puis à nouveau évoquées dix ans plus tard, en 1889. L'initiative est venue d'un négociant retraité, Joseph Victor Barbier (1840-1898), qui, doté d'une formation initiale de dessinateur, s'était passionné pour la cartographie. Au cours des contacts qu'il prend afin de faire connaître ses cartes, il conçoit le projet de créer à Nancy une société de géographie, sur le modèle de celles qui se sont constituées en 1873 à Lyon et en 1874 à Bordeaux. Il n'en existe encore aucune à cette date dans la moitié Nord de la France, à l'exception bien entendu de la société mère parisienne, qui remonte à 1821 . Il provoque en décembre 1878 une première réunion privée, à laquelle sont surtout conviés des universitaires et quelques personnalités particulièrement intéressées : les facultés des Lettres, des Sciences, de Droit sont représentées par leurs doyens, l'École forestière par son directeur. Lors de la réunion suivante, on élargit le comité en y faisant entrer des notables élus, des ingénieurs, des représentants du commerce et de l'industrie. Le succès est grand dès le début: 445 membres souscripteurs se font inscrire, alors qu'on en attendait environ 300. Les militaires en activité ne sont pas autorisés à adhérer à la société de géographie, ils ne le seront que dix ans plus tard. En attendant, et pour bien montrer l'intérêt qu'on attache à leur soutien, c'est un officier retraité, le chef d'escadrons de Carcy, qui est désigné comme président du comité provisoire. La promotion de la géographie, dans les années qui suivent la défaite française de 1870-1871, est considérée comme une œuvre patriotique éminente.

Fig. 1 : Évolution des effectifs de la Société de Géographie de l'Est

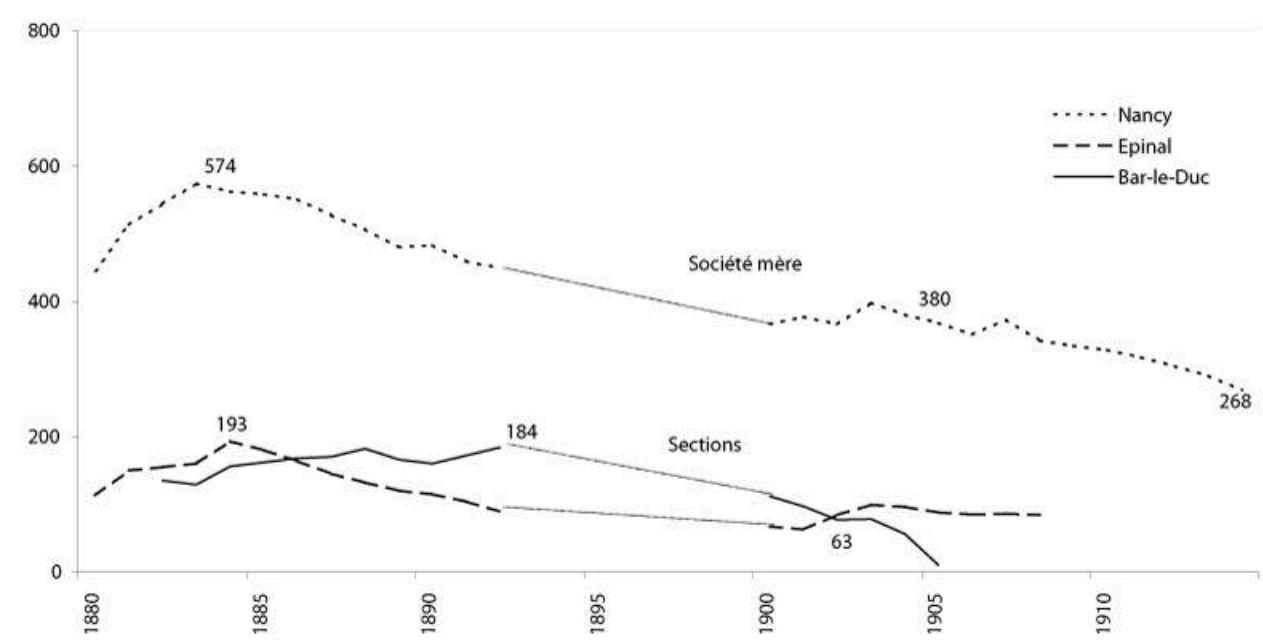

Il s'agit du nombre des membres souscripteurs, les seuls reflétant réellement la vitalité de la société. 
5 L'impulsion donnée à Nancy se communique rapidement dans les chefs-lieux des deux autres départements lorrains. Une section est constituée d'abord à Épinal, où l'on recrute un nombre d'adhérents dépassant la centaine. L'effectif maximum est atteint en 1883, avec 193 souscripteurs. Cela coïncide évidemment avec les années où Jules Ferry dirige la politique française et lance la France sur la voie de multiples interventions coloniales. Mais la section manque de dynamisme, ses effectifs s'effilochent rapidement; la ville est mal placée pour attirer les conférenciers, qui ne peuvent prendre le temps d'aller jusqu'à Épinal, lorsqu'ils viennent à Nancy. À la fin de 1893, il devient nécessaire de renouveler entièrement son comité : on y fait entrer plusieurs industriels très en vue et un banquier. "Nous avons eu la conviction, écrit alors J.V.Barbier, que dans un centre industriel et commercial comme Épinal, non seulement la géographie doit tenir une très grande place, mais encore que l'industrie devait tenir une grande place dans une société de géographie ». Les industriels cotonniers, dont les sources d'approvisionnement, les débouchés et les concurrents se trouvent outre-mer, acceptent de participer à cet effort de relance, qui ne porte pas ses fruits immédiatement. Après le point le plus bas touché en 1900, avec 63 adhérents, une remontée significative se produit dans les premières années du siècle. En 1908, la section vosgienne se sent suffisamment forte pour prendre son indépendance vis-à-vis de la société mère et pour constituer une société de géographie autonome.

6 Créée seulement en janvier 1881, la section meusienne fait longtemps preuve d'une plus grande vitalité. Le nombre des souscripteurs augmente régulièrement et atteint un maximum de 184 en 1891. Le dynamisme de la section de Bar-le-Duc s'explique d'abord par l'inlassable activité de son fondateur, l'imprimeur Claude Bonnabelle, mais aussi par la plus grande facilité de faire venir des conférenciers passant par Nancy, grâce à la gare située sur la voie ferrée Paris-Strasbourg. On peut croire aussi que les appuis locaux ont été plus énergiques qu'à Épinal, et la société mieux implantée dans l'ensemble $\mathrm{du}$ département que dans les Vosges. La création d'un «musée ethnographique, colonial et industriel», inauguré en 1891, manifeste l'impact important de la société dans la ville de Bar-le-Duc. Mais à l'inverse d'Épinal, on voit une érosion des effectifs apparaître dans les années 1890 et s'accentuer gravement après le décès de Bonnabelle (1896). Rien ne peut arrêter l'effondrement et on voit la société disparaître définitivement en 1904. Ses adhérents vont alors rejoindre les autres sociétés savantes du département de la Meuse auxquelles ils appartenaient déjà.

7 À Nancy, après un très vif engouement, qui porte l'effectif maximum des souscripteurs à 574 en 1882, on assiste à une décroissance lente et régulière. À la mort de Barbier (1898), la société reçoit tout de même encore la cotisation de près de 400 personnes. La société reste très vivante. Les conférences sont nombreuses et intéressantes ( 6 à 10 par an). Le secrétaire général J.V. Barbier ne ménage pas sa peine, lors de ses déplacements à Paris ou à l'occasion des congrès auxquels il participe, pour contacter les explorateurs et les voyageurs les plus connus et pour sélectionner parmi eux les meilleurs orateurs. C'est encore lui qui veille à les accueillir et à les héberger à Nancy dans des conditions qui les incite à y revenir. Le public reste fidèle dans son ensemble et, lors des conférences exceptionnelles, on se plaint même de l'afflux excessif des "invités ", qui ne sont pas membres de la société. Si l'on assiste à une baisse continue des adhésions, c'est à cause des décès et des départs, qui ne sont pas compensés par l'arrivée de nouveaux membres. C'est aussi la conséquence d'une certaine évolution des mœurs à Nancy : les femmes ne s'intéressent guère à ce type d'activité et souvent finissent par 
dissuader leurs maris d'y participer; dans les familles, on fait passer son bien être avant tout le reste et l'on vit de manière plus égoïste qu'autrefois; dans les années de crise économique que l'on connaît alors, chacun limite ses dépenses et tend à se replier sur lui-même.

Mais, contrairement à ce qui s'est produit à Bar-le-Duc, la mort du fondateur n'entraîne pas l'effondrement de la société. Elle connaît au contraire un rajeunissement salutaire, que nous caractériserons plus loin, lorsqu'il sera question de l'évolution du bulletin. De 1900 à 1906, les effectifs se stabilisent sur un palier, vers 370-380. Puis la situation se dégrade rapidement dans les années qui précèdent immédiatement la guerre : il ne reste plus que 268 souscripteurs en 1913. Les préoccupations des Lorrains ont changé. La large ouverture sur le monde qu'avait apportée la géographie avait joué le rôle d'un dérivatif et réduit l'amertume de la défaite de 1871. Les plus généreux avaient même rêvé d'un monde ouvert, dans lequel les rivalités commerciales remplaceraient à jamais les conflits militaires et dans lequel tous les grands pays collaboreraient dans un même souci du progrès scientifique. Mais au cours des années 1905-1910, on voit cet état d'esprit disparaître progressivement. Les menaces anciennes sont de retour, et elles se manifestent en Europe, à la frontière même de la France. Comme d'autres institutions, la société de géographie de Nancy courbe la tête devant la tempête qui approche.

\section{L'apport du bulletin de la société à la géographie française (fig. 2)}

9 Dès l'origine, sans doute pour montrer la grande extension du champ de la géographie, et peut-être aussi pour se dispenser d'une sélection plus rigoureuse, J.V. Barbier classe les articles publiés dans son Bulletin sous un certain nombre de rubriques. Cette classification, souvent très arbitraire comme nous le verrons, est restée en vigueur jusqu'en 1899 ; elle a disparu à partir de l'année 1900.

\section{A. Une classification arbitraire et une répartition déséquilibrée}

10 La géographie militante, considérée comme plus noble par les sacrifices qu'elle implique, est toujours citée en premier. Elle fait place essentiellement aux récits des explorateurs et des voyageurs qui ont parcouru des régions encore mal connues. Leurs carnets, souvent écrits de manière vivante et pittoresque, constituent un genre littéraire dont le public est très friand. Ces longues relations sont découpées entre les numéros, pour constituer de véritables feuilletons qui se poursuivent parfois pendant plusieurs années. Quand le récit a beaucoup de succès, il arrive que la maison BergerLevrault se charge de le publier sous la forme d'un volume entier. 
Fig. 2 : Évolution du nombre de pages du Bulletin de la Société de Géographie de l'Est, entre 1879 et 1913

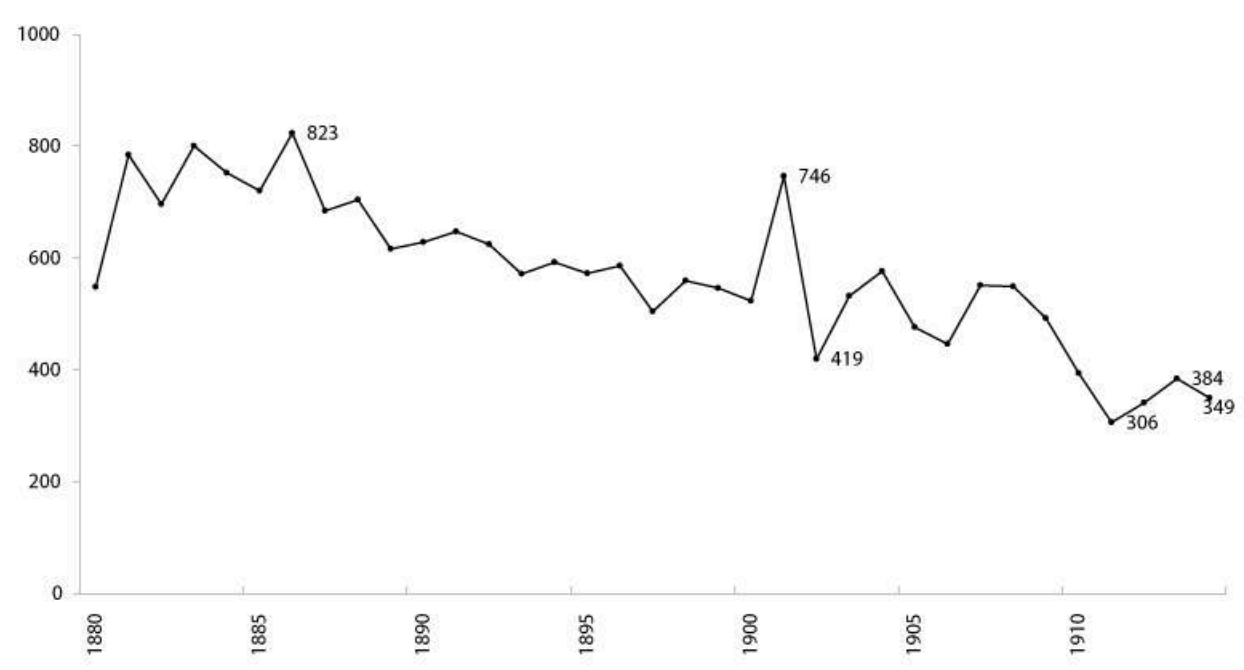

Pour permettre une meilleure comparaison entre les années, on a exclu les listes de membres (30 à 40 pages), qui sont ou ne sont pas paginées normalement et ne figurent pas dans le bulletin de toutes les années. En 1899, il est clair que le comité de rédaction a voulu marquer spécialement les 20 ans de la revue ; cette remontée éphémère de la pagination n'a pas eu de lendemain.

11 Il n'existe qu'une différence très mince entre la géographie militante et la géographie coloniale. En principe, la seconde devrait ne s'intéresser qu'aux territoires déjà suffisamment connus, ayant reçu un début d'équipement et une première installation de colons. Dans la réalité, la distinction n'est pas toujours bien claire, ni respectée. On classe dans la géographie militante une expédition de style militaire en Tunisie (1883-1885), alors qu'à la même époque, des relations de séjour au Congo avec Brazza ou des notes sur Madagascar sont classées dans la géographie coloniale. Quant à la géographie dite commerciale, elle n'est en fait qu'un appendice de la géographie coloniale. C'est une rubrique particulièrement difficile à remplir : les hommes d'action n'ont pas le temps de faire de longs discours. Quelques conférences isolées, quelques notes d'attachés commerciaux ou de négociants insistent sur les possibilités commerciales qu'offrent à la France les territoires nouvellement colonisés.

Dans toutes ces matières où le public est particulièrement demandeur, il est difficile d'avoir l'exclusivité de bons textes à publier. Pour alimenter ses rubriques, J.V. Barbier doit faire appel à un réseau de correspondants plus ou moins fidèles, rencontrés à l'occasion d'un congrès ou ayant des attaches avec la Lorraine. Les marins sont à l'époque ceux dont les préoccupations paraissent les plus proches de celles des géographes. Le docteur Hagen, médecin de la marine, fournit des indications très précieuses sur les Nouvelles-Hébrides, en insistant particulièrement sur le climat, la situation sanitaire, l'hygiène et le mode de vie des populations. E. Courtet sert dans l'artillerie de marine successivement à Tahiti, en Indochine, puis à Madagascar. Sa démarche est au début celle d'un ethnographe et d'un linguiste, mais il donne ensuite de la vie à Madagascar une description très concrète, fondée sur sa propre expérience, qui contredit quelque peu l'enthousiasme officiel. Payeur-Didelot est un cas intéressant : il est le seul survivant de la mission Crevaux, en Amérique du Sud. C'est un expatrié, qui a quitté la Lorraine pour échapper à l'incorporation dans l'armée allemande ; à ce titre, il est représentatif du réseau d'Alsaciens-Lorrains qui s'est mis en 
place un peu partout dans le monde, après 1871. En 1883, à l'âge de 19 ans, il s'engage dans la mission de Brazza en Afrique centrale. Il confie au Bulletin une longue étude sur le Congo-Gabon, qui commence comme un simple carnet de route, mais se continue par des études beaucoup plus détaillées. Un autre exemple caractéristique est celui de Paris : ce membre correspondant de la Société est agent des postes et télégraphes à Hanoï. À ce titre, il est chargé de construire des lignes à travers l'Annam et le Tonkin. Il vit au milieu des indigènes, et ses fonctions le mettent à même de bien décrire le mode de vie des populations. Nous citerons encore la contribution d'un industriel vosgien, Ancel Seitz, «Le Congo français au point de vue commercial » : elle montre comment chacun peut enrichir la science géographique de son expérience professionnelle.

Dans tous ces travaux, certaines parties du monde risquent d'être un peu oubliées. Pour les pays d'Asie centrale, on fait appel à des traductions de travaux russes. Pour d'autres pays, on peut mettre à contribution les comptes-rendus des membres de la Société qui ont eux-mêmes accompli des voyages : Charles Grad raconte une excursion au Sinaï, P. Collesson un circuit en Scandinavie.

Mais c'est surtout J. Thoulet qui se révèle un observateur remarquable, au cours de son voyage de six mois à Terre-Neuve. Nous reviendrons plus loin sur ce que ce récit nous apprend de la personnalité scientifique de son auteur.

15 La Géographie régionale est le deuxième pilier sur lequel reposent les sociétés de géographie, qui ont toutes une base régionale. Leurs adhérents ne sont pas seulement attirés par les aventures coloniales et lointaines, ils veulent aussi une géographie de proximité, qui les instruise sur le pays dans lequel ils vivent. Cependant, cette approche est déjà celle des sociétés savantes régionales, qui connaissent un regain de faveur après 1870 : il ne peut être question de leur faire simplement concurrence, les Sociétés de Géographie doivent obligatoirement apporter quelque chose de nouveau.

Cela justifie l'ouverture d'une rubrique de géographie "régionale». Mais que faut-il entendre au juste par ce terme? Le flottement est total. J.V. Barbier a la fâcheuse tendance à baptiser géographie «régionale» toute étude, quelle qu'elle soit, intéressant la région de l'Est. Lorsque Auerbach en 1890 s'attaque à une description géographique du Plateau lorrain, il introduit en France le vocable de " chorographie ", ce qui lui permet de prendre ses distances vis-à-vis de Barbier et de souligner tout ce que sa démarche a de réellement scientifique.

17 Les contributions de qualité sont extrêmement rares dans les premières années. Les articles sur le pays de Dabo et le Donon sont traités dans le style de descriptions pittoresques, qui ne peuvent intéresser que les touristes. La toponymie tient une grande place dans le Bulletin, avec tout ce qu'elle peut comporter de hasardeux, lorsqu'elle est maniée par des non-linguistes. À côté d'une assez bonne mise au point de Charles Grad sur le Rhin, le seul article qui émerge vraiment dans cette première période est l'excellent exposé de géographie historique de l'historien universitaire Christian Pfister sur la limite des langues française et allemande en Alsace et en Lorraine (1890).

18 Un certain renouvellement semble pouvoir provenir des monographies qui ont été demandées aux instituteurs des départements pour l'Exposition Universelle de 1889. La Société s'est beaucoup impliquée dans leur réalisation aux côtés de l'inspecteur d'Académie. Elle en publie quelques-unes sous la forme de résumés en 1891 et 1892. Sans doute ont-elles pour intérêt de favoriser la pénétration de la Société dans l'enseignement public. Mais ces notices sont décevantes; elles ont été rédigées par des 
instituteurs qui n'ont reçu aucune formation géographique véritable ; elles manquent d'esprit de synthèse et d'homogénéité.

Une dizaine d'autres rubriques sont utilisées dans le Bulletin, de manière parfois épisodique. Elles contribuent à créer une impression de désordre, sous un ordre apparent. On ne comprend pas bien pourquoi la géographie économique est séparée de la géographie commerciale. Mais ce qui est produit sous ce nom est à peu près inexistant. L'article du baron Hulot, qui présente le Canada de l'Atlantique au Pacifique, est un récit de voyage peu différent des autres, mais qui met seulement l'accent davantage sur la nature des moyens de communication utilisés. La longue conférence de Paul Macey, chargé de mission pour le Syndicat français du Haut-Laos, aurait évidemment dû trouver sa place dans la géographie coloniale: mais il montre qu'il s'agit dans ce pays d'une colonisation économique, donc totalement pacifique ; c'est ce qui explique le choix de la rubrique! Un article de Guénot, de la Société de Géographie de Toulouse, sur le déboisement des Pyrénées, n'a strictement rien à voir avec l'économie. Autre objet de confusion: la Géographie rétrospective et historique. On nous présente sous ce titre, tantôt de courtes notes relatives à l'histoire de la géographie, tantôt des récits de voyages restés une quarantaine d'années dans un tiroir, tantôt des travaux qui relèvent de l'archéologie ou de la promenade archéologique. Mais le plus beau contresens est sans contestation possible celui qui s'attache à la Géographie générale. Elle est représentée par deux travaux qui n'ont évidemment rien à voir avec elle et qui trouveraient place facilement eux aussi dans la géographie coloniale: une étude sur les Russes et l'Asie centrale, et une longue description, par le capitaine de vaisseau en retraite H. Jouan, des îles de l'Océanie. Ce dernier travail, de caractère surtout encyclopédique, est l'œuvre d'un naturaliste et d'un ethnographe.

20 Il n'y a pas grand chose à dire de la géographie mathématique, qui recouvre la cartographie, spécialité dans laquelle Barbier est plus à l'aise, ni de la géologie militaire, qui n'a fait l'objet que d'un seul article. Il était intéressant d'introduire une rubrique de Géographie politique: elle fait l'objet d'une unique contribution, de Perdrizet, et traite encore d'un problème colonial: le conflit anglo-hollandais en Guyane. Sous la rubrique Géographie scientifique, on relève sur la rade de Brest un article qui s'apparente à une explication de carte géologique. Enfin, on notera que la Géographie physique, réduite à la portion congrue, est la seule à ne pas être polluée par des emprunts à des domaines étrangers : elle se limite strictement à l'Océanographie, dont Thoulet est à cette époque en France le meilleur représentant.

\section{B. Un bulletin mieux ordonné et plus scientifique}

Avant même le décès de Barbier, Thoulet publie, dans le bulletin de la société de l'année 1895 , sous le titre "Les sociétés de géographie et la décentralisation", une critique virulente, mais particulièrement fondée, de leurs pratiques. Bien qu'il s'en défende, elle vaut évidemment pour celle de Nancy comme pour les autres. Il met en cause l'orientation générale et le choix des articles. Leurs revues donnent en abondance les comptes-rendus d'expéditions menées dans les diverses parties du monde : mais ce sont des textes trop prolixes, bourrés de renseignements anecdotiques dépourvus d'intérêt scientifique ; souvent ils ont déjà été publiés ailleurs et ils arrivent toujours trop tard, quand le public a été déjà informé des événements qu'ils relatent par la lecture de la presse quotidienne. Elles font aussi la place trop belle aux conférences, qui 
ne sont pas des travaux originaux. En outre, Thoulet trouve anormal que chaque société traite du monde entier et s'arroge une compétence universelle. Elles devraient laisser cette prérogative à la société parisienne et accorder, chacune en ce qui la concerne, plus de place à la connaissance de leur propre région : "d'abord la région, puis le globe, s'il reste de la place dans le volume». Le respect de cette règle simple permettrait un meilleur classement des travaux géographiques, que l'on pourrait retrouver de manière plus sûre.

Ces réflexions critiques, salutaires et émanant d'un scientifique qui avait soutenu dès le début l'action de la société, ne donnèrent pas de résultat immédiat, mais furent tout de même bien accueillies. Dans son rapport annuel du début de l'année 1896, Barbier fait état de la constitution de commissions, chargées de définir de nouvelles orientations. L'une s'occupera du classement de la bibliographie lorraine, une autre traitera de la vulgarisation des renseignements coloniaux. Mais la principale nouveauté, dans l'immédiat, réside dans l'organisation d'excursions à la belle saison. Elles ont pour but, en la présentant sous un jour agréable, de faire découvrir en Lorraine des sites naturels, historiques ou industriels que les habitants connaissent mal, et dont ils ignorent les transformations récentes. Bien préparées, suivies par des groupes de 30 à 50 personnes, les excursions constituent, de 1896 jusqu'à la guerre, une des activités les plus appréciées de la société de géographie. Pfister et Bleicher montrent les premiers par l'exemple qu'on peut leur donner un contenu scientifique tout à fait appréciable. Elles sont consacrées à des sites lorrains, accessibles en chemin de fer ou en voitures particulières, elles combinent surtout promenades géologiques, visites de monuments historiques et enquêtes sur des activités économiques régionales.

Une deuxième étape de la rénovation s'affirme en 1900, lors de la vingtième année du bulletin. Cette fois, c'est toute la présentation qui est modernisée et rendue plus rationnelle. L'ancien système des rubriques est totalement supprimé. Les articles sont sélectionnés avec plus de soin. Dans le cadre d'un bulletin dont le nombre de pages diminue sans cesse, les articles deviennent plus courts, car plus condensés; leur longueur est proportionnelle à la densité des informations qu'ils renferment. Ils alternent avec les comptes-rendus des conférences les plus intéressantes. Pourtant, les informations contenues dans le cadre des "Miscellanées", qui sont une sorte de revue des périodiques, et dans le cadre des « Faits géographiques ", qui sont une chronique de l'actualité, demeurent pratiquement inchangées et occupent toujours autant de place. C'est dire que proportionnellement, leur part se trouve renforcée dans le bulletin. Elles sont le lien qui soude une communauté de lecteurs et qui fait prendre conscience de l'appartenance, en dépit des diversités de situation, à la grande famille des géographes. En outre, le vœu de Thoulet ne peut être entièrement exaucé : les articles relatifs à l'Est de la France ne sont pas encore très nombreux.

Quelques articles émergent de cette période, dont certains sont l'expression de véritables travaux universitaires. En 1898, déjà, un des premiers élèves d'Auerbach, $\mathrm{E}$. Audriot, professeur au collège de Lunéville, donne une intéressante étude de géographie régionale, explicative et raisonnée: «Répartition de la population dans l'arrondissement de Lunéville, d'après le relief, la nature du sol, les cultures, les industries ». En 1900, Gustave Bleicher publie une bonne monographie du «Plateau central de Haye ", où il a conduit précédemment une excursion. Lucien Gallois, maître de conférences à l'École normale supérieure, inaugure une série d'articles célèbres sur la géographie à Saint-Dié à l'époque de la Renaissance. En 1901, le même Bleicher livre 
encore, avec l'autorisation des Annales de géographie, l'article célèbre et un peu malheureux, où il met en doute un passage récent de la Moselle par le Val-de-l'Âne. Jean Thoulet apporte une contribution sur «le fond de la mer ». En 1904, le même Thoulet produit le texte d'une conférence faite en Sorbonne: «Les lois physiques de l'océan ». En 1905, un polytechnicien, A. Nérot, communique le rapport qu'il a adressé à la Société industrielle de l'Est sur «le Nord et l'Est de la France et les voies d'accès au Simplon ». En 1906, Émile Chantriot commence une série de grande valeur scientifique sur « les cartes anciennes de la Champagne ». L'année 1908 voit Auerbach poser en des pages très brèves la question des projets de canalisation de la Sarre, de la Moselle et de la Chiers, tandis que Thoulet traite de la transparence des eaux. En 1910, Henry Joly souligne le rôle de la tectonique dans les recherches de houille en Meurthe-et-Moselle. En 1911, le bulletin offre l'hospitalité à une courte note de Paul Vidal de la Blache : « de l'interprétation géographique des paysages ». À ces articles marquants, qui sont souvent assez courts, s'ajoutent encore quelques travaux d'érudits régionaux, des comptes-rendus de conférences et des études, généralement sérieuses, sur des pays lointains. L'ensemble atteint un niveau que l'on peut juger très honorable.

\section{Une pléiade de savants, qui se réclament de la géographie} empiéter sur le domaine de la géographie physique, dont il reconnaît pourtant l'utilité. Il accepte volontiers que cette dernière soit enseignée à la faculté des sciences et engage même ses étudiants à en suivre les cours. Il se consacre entièrement à la géographie régionale, puis à la géographie humaine et économique. Homme de dossiers plutôt que de terrain, il aime classer les ouvrages de la bibliothèque de la société et se tient fidèlement au courant de toutes les productions allemandes. Il est particulièrement sensible à tout ce que la géographie peut attendre des progrès de la statistique, dont il montre qu'on peut manier les chiffres avec intelligence. Après la mort de Fliche, en 1908, Auerbach devient président de la société. Nous n'insisterons pas davantage ici sur ce personnage attachant, auquel nous avons déjà consacré une publication et qui fait l'objet d'un article dans le présent numéro de la Revue géographique de l'Est. À partir de 1906, un deuxième géographe fait son apparition : il s'agit d'Émile Chantriot, professeur au lycée de Nancy et à l'École supérieure de commerce. Auteur d'une thèse de géographie régionale sur la Champagne, il apporte surtout une contribution dans le domaine de la géographie commerciale.

27 L'histoire fournit dans le comité un appoint important. Cela est logique à une époque où, dans l'enseignement secondaire et même supérieur, elle est encore très mal séparée de la géographie. Jusqu'à son départ pour Paris, elle est surtout représentée par Christian Pfister, président de la société de 1893 à 1902. Ce maître incontesté a su, dans 
des ouvrages qui font encore autorité aujourd'hui, dessiner de manière très claire les traits de la personnalité historique de Nancy et de la Lorraine. Il se rapproche particulièrement des géographes lorsqu'il s'intéresse à l'histoire des lieux et par son sens du cadre spatial dans lequel se déroulent les événements historiques. Son étude de la limite linguistique du français et des parlers germaniques en Lorraine est considérée comme un modèle. Mais un autre historien se révèle encore plus proche que lui de la géographie moderne : c'est Charles Guyot, professeur de droit à l'École forestière. À travers ses études de l'histoire des forêts lorraines, mais aussi de la vie rurale dans son ensemble et du peuplement, il apparait comme un spécialiste de l'histoire des paysages et un précurseur de la géographie historique.

En face de ces historiens et des géographes littéraires, le poids des scientifiques paraît écrasant. Malgré la présence à Nancy de l'École forestière, la botanique n'apporte à la géographie qu'un appoint assez limité. Paul Fliche (1836-1908), qui la représente principalement, a été élève de Godron, puis professeur d'histoire naturelle avec différents titres de 1865 à 1902. Il a présidé la société de géographie de 1902 à 1908. Mais ses orientations personnelles le portent plutôt vers la géologie et l'histoire: spécialiste des flores fossiles, il collabore avec les paléontologues et les préhistoriens. Il s'intéresse donc peu à la géographie botanique, qui est en train de naître à cette époque en France avec Charles Flahault, soutenu par les Annales de Géographie. Mais celle-ci est tout de même enseignée à Nancy par E. Gain, qu'on a chargé de la direction de l'institut colonial, où il s'est assuré la collaboration d'Auerbach. Il y enseigne notamment une botanique coloniale appliquée, assez proche des préoccupations des géographes, et publie en 1908 ses conférences sous le titre «Introduction à l'étude des régions florales $"$.

Jean Thoulet, né en 1843 à Alger, mériterait à lui seul un long article, car il représente une personnalité de premier plan. Véritable fondateur de l'océanographie française, il étudie d'abord les lacs suisses, puis participe à une longue mission océanographique à Terre-Neuve. Il est en relations étroites avec le prince de Monaco et considéré comme un expert de classe internationale. Après son arrivée à Nancy, il est officiellement chargé, en 1892, du cours de " géographie physique » qui est alors créé à la faculté des Sciences. Il y enseigne surtout l'océanographie. Il est donc le pendant d'Auerbach, et les étudiants ne peuvent se dire géographes que s'ils ont suivi à la fois le cours d'Auerbach et celui de Thoulet. Il est important de constater qu'à Nancy il n'y a pas eu, au moins au début, d'impérialisme de la géologie sur la géographie physique. Alors que les géologues restreignent souvent les perspectives de la géographie à une géomorphologie réduite à une confrontation du terrain et de la carte, Thoulet ouvre au contraire de larges horizons vers une géographie physique globale, fondée sur l'expérimentation et sur des mesures précises, plus proche des sciences physiques que des sciences naturelles. Dépassant le cadre régional, il situe la réflexion géographique dans une dimension planétaire. Il est dommage que son œuvre n'ait pu être poursuivie à Nancy, après son départ à la retraite en 1913. Avec René Nicklès et Joly, ce sont les géologues qui vont prendre le dessus et orienter pour longtemps une grande partie de la production géographique lorraine vers l'étude du relief régional et vers celle des activités minières.

Gustave Bleicher représente longtemps les géologues dans l'équipe de la société de géographie de l'Est. Mais c'est un géologue plutôt atypique. Sa formation est celle d'un naturaliste au sens large, ce qui le prédispose à une plus grande ouverture vers toutes 
les formes de géographie. Il est issu de l'école de santé militaire de Strasbourg, où il a obtenu son doctorat en médecine. Tout en exerçant sa profession, il occupe ses loisirs à préparer une thèse de géologie, qui lui vaut en 1870 le doctorat ès sciences. Cette thèse l'oriente particulièrement vers l'explication du relief : il s'intéresse à la façon dont les couches sédimentaires autrefois présentes sur le sommet des montagnes ont été décapées par l'érosion et transportées par les anciens cours d'eau sur les plateaux situés en contrebas. Le massif vosgien et le plateau lorrain permettent d'illustrer particulièrement ce thème. À sa retraite, en 1876, Bleicher postule pour une chaire d'histoire naturelle : elle lui est accordée à l'école supérieure de pharmacie de Nancy. Sans cesser de s'intéresser à la géologie, il se consacre alors à la flore, à la faune de la région, ainsi qu'à l'archéologie préhistorique. Le livre qu'il publie en 1889: «Les Vosges, le sol et les habitants » montre bien son éclectisme. Avec un esprit scientifique rigoureux, il apporte sur le milieu physique vosgien et sur les populations de cette montagne des vues originales et solides. On y trouve toute la matière d'une excellente géographie. Un géographe pourrait toutefois lui reprocher, outre certaines digressions, de ne pas avoir assez mis en valeur les relations de causalité entre des phénomènes d'ordre divers, que le scientifique sépare pour les étudier, mais que le géographe doit réunir.

31 Les témoignages qui s'expriment à la mort de Bleicher, assassiné dans son laboratoire en juin 1901, insistent sur le fait que ce maitre avait un rayonnement remarquable. Plus que d'autres, il a formé des disciples, qui ont été des ingénieurs et des savants : parmi eux, on cite alors le géologue René Nicklès et surtout le docteur Imbeaux, hydrologue, ingénieur de la ville de Nancy. Ce dernier est l'auteur d'une thèse d'hydrologie continentale sur la Durance, dans laquelle il s'efforce d'appliquer, à plus de trente ans de distance, la méthode de Belgrand sur la Seine. Il est le pionnier de cette discipline, qui complète bien l'océanographie de Thoulet et la climatologie de Millot.

Le moment est venu de rendre hommage à Charles Millot, que nous n'avons pas encore évoqué. Il n'a jamais voulu occuper un rang honorifique dans la société, se contentant d'être quelque temps vice-président puis seulement vice-président d'honneur. Mais il a été à côté des secrétaires généraux la véritable cheville ouvrière de tous les bulletins. C'est lui qui a patiemment collectionné les extraits d'articles avec lesquels il a composé les Miscellanées et qui a tenu une chronique fidèle et prodigieusement bien informée des Faits géographiques recueillis sur les cinq continents. Si l'on mettait bout à bout, en les classant par thèmes, tous les textes de Millot, on obtiendrait sinon un traité complet de géographie, du moins un manuel de culture géographique. À une époque où chacun en Lorraine tourne les yeux vers la science allemande, sa connaissance parfaite de l'anglais lui permet de traduire et de faire connaitre beaucoup de publications anglosaxonnes. Il sait aussi découvrir, dans les publications les plus diverses, des informations originales qui enrichissent notre vision géographique du monde.

Mais Charles Millot a joué aussi un rôle capital par son enseignement. Né à Nancy en 1847, il est entré à l'École navale et a mené à partir de 1865 la vie d'un officier de marine, qui a sillonné toutes les mers du monde et a effectué toutes sortes de travaux hydrographiques. En 1878, il démissionne de la marine, avec le grade de lieutenant de vaisseau. Il devient alors disponible pour prendre la direction de la Commission départementale de météorologie. Il participe dès l'origine à la fondation de la société de géographie de l'Est et rassemble dès la première année des nouvelles géographiques. D'abord chef de travaux, il est chargé en 1884 d'un cours complémentaire de 
météorologie à la faculté des Sciences de Nancy. Il est destiné aussi « aux étudiants de la faculté des Lettres qui veulent aborder l'étude de la géographie physique sans connaissance suffisante des sciences physiques et naturelles». Ce cours est d'abord polycopié, puis publié en 1901 sous le titre "Notions de météorologie utiles à la géographie physique ». Il y fait notamment référence aux observations menées en Lorraine soit par les forestiers, soit par lui-même et s'efforce de donner chaque fois une explication complète des mécanismes. Charles Millot a publié également, dans le bulletin de la société de géographie, plusieurs articles de valeur consacrés au climat de Nancy et de la Lorraine. On citera particulièrement « la pluie à Nancy, 1878-1907 » dans le bulletin de 1908.

\section{La construction d'une géographie scientifique et rigoureuse}

Pour la géographie française, le problème à résoudre est de taille : comment faire émerger une discipline nouvelle, qui est mal enseignée, et qui n'est encore représentée que par une petite poignée de spécialistes? Il faut à la fois la rendre populaire, démontrer son utilité, l'ouvrir largement vers toutes les sciences, mais en même temps définir ce qui fait sa valeur et son originalité. Le modèle existe : on le trouve dans la géographie allemande, qui s'est déjà constituée en discipline rationnelle et a obtenu d'être reconnue. Mais dans le public de la France de l'Est, il ne s'impose qu'avec lenteur.

\section{A. Une définition encore très floue de la géographie (1880-1890)}

Faute de savoir encore exactement ce qu'elle est, ni davantage ce qu'elle n'est pas, la géographie de 1880 se montre extrêmement accueillante. Toutes les sciences physiques et naturelles, par un côté ou par un autre, touchent à la géographie et peuvent lui apporter une contribution; l'histoire pure est la bienvenue, lorsqu'elle s'intéresse à un peuple, à une région ou à un site particulier; la linguistique trouve sa place, lorsqu'elle s'applique aux noms de lieux; les récits de voyage donnent une vision préscientifique, tantôt exacte, tantôt romancée, des lieux encore mal connus. Partout la géographie trouve du grain à moudre et cela aboutit parfois à des compilations composées sans grand esprit critique. Ce laxisme sympathique trouve son expression particulière dans "Le livre d'or de la géographie dans l'Est de la France », un florilège où J.V. Barbier passe en revue les missionnaires, les commerçants, les diplomates, les voyageurs, les aventuriers et les rares géographes dont on a gardé la mémoire (1880-1882).

36 Si ici et là, en France, des éléments de débats commencent à apparaître au sujet de ce que doit être la géographie, ils ne semblent pas avoir marqué la société de Nancy, qui vit jusqu'au milieu des années 1890 sur l'idée qu'il faut vulgariser, sans se demander s'il est sensé de vouloir vulgariser dans le public une science qui n'existe pas encore.

\section{Une conception encyclopédique}

Cette conception, qui est celle de Barbier et d'un grand nombre de membres, est développée de manière très nette par le général Hanrion, président de la société, à l'assemblée générale du 12 février 1891. Il définit la géographie comme "étude et connaissance de la terre sous tous ses aspects». Envisagée de cette façon, elle se 
rattache à presque toutes les branches du savoir humain et doit emprunter ses notions principales à de nombreuses sciences. Il en résulte inévitablement une situation de dépendance, en même temps qu'un émiettement total: on distingue une géographie physique, une géographie politique, une géographie mathématique, des géographies agricole, minérale, industrielle, commerciale, etc. Ce sont, dit-il, autant de cas particuliers d'une même science, qui s'appelle d'un nom générique «la géographie ». Barbier ne disait pas autre chose en 1881: «La géographie n'est pas une science par elle-même, c'est la convergence de toutes les sciences naturelles et mathématiques vers le but commun: la connaissance du globe. Un géographe n'est pas un savant, c'est plusieurs savants réunis en un seul ». Il n'est guère besoin de souligner la faiblesse d'une telle conception: en définissant la géographie par son objet et non par une méthode, on en fait une science caméléon, obligée d'adapter sans cesse ses moyens d'approche aux faits qu'elle étudie.

\section{Le désir d'une géographie utilitaire}

On voit s'affronter, au cours de ces premières années, les partisans d'une géographie théorique et scientifique et ceux d'une géographie utilitaire. Lors du Congrès des sociétés françaises de géographie, tenu à Nancy en 1880, Henry Bionne, partisan de la "géographie active» (sic), s'oppose à Levasseur, qui privilégie l'enseignement. La géographie "commerciale », c'est-à-dire appliquée au commerce et à l'industrie, fait une percée. Barbier s'en réjouit, et trouve même que le congrès fait une trop grande place à la géographie scientifique, qu'il faudrait réserver pour des réunions spéciales. Marcel Dubois, qui devient quelque temps professeur à la faculté des lettres, approuve cette orientation. En 1884, la société engage des pourparlers avec la Chambre de commerce de Nancy, en vue de la création d'un cours de géographie commerciale, non dans un lycée, mais dans une école d'enseignement professionnel. Ils n'aboutissent pas, mais Marcel Dubois, de sa propre initiative, fonde un cours de géographie commerciale à l'École professionnelle de l'Est (rapport annuel du 30.1.1885). La section meusienne de la société, qui dispose d'une bibliothèque et d'un musée, veut être en 1889 « un centre de renseignements pour le commerce d'importation et d'exportation». Tout naturellement, les sociétés de géographie françaises s'intéressent à la création de bureaux de renseignements coloniaux, destinés à informer les jeunes gens qui veulent aller vivre aux colonies. Celle de Nancy salue avec une particulière satisfaction, en 1897, la naissance de l'École supérieure de commerce de Nancy, en raison des relations étroites qui existent entre commerce et géographie. Elle est destinée à apporter à de jeunes bacheliers les connaissances nécessaires à la pratique des affaires, ainsi qu'à former des agents consulaires, qui représenteront les intérêts économiques français à l'étranger.

Dans les dernières années du siècle, l'engouement pour cette géographie utilitaire ne cesse de monter partout. Thoulet, qui rend compte du Congrès des sociétés françaises de géographie, tenu à Marseille du 18 au 25 septembre 1898, s'en alarme : «Dans ces réunions où chaque ville plaide pro domo sua, la science pure qui possède parmi nous une maison - hélas - de plus en plus restreinte, perd quelque peu de ce que nous avons été élevés à considérer comme ses droits. Si quelques personnalités en montrent encore le souci et exposent des travaux dépourvus en apparence d'applications immédiates, elles doivent s'estimer heureuses d'être écoutées par un auditoire bienveillant. On n'ose demander davantage à une époque d'utilitarisme à outrance, où 
l'on vit comme s'il n'y avait plus ni veille, ni lendemain, comme si l'heure présente et peut-être la minute qui suit immédiatement existaient seules ». Et il ajoute : lorsque la fièvre actuelle sera passée, on comprendra que le présent n'est pas tout.

\section{Le désir d'une meilleure connaissance de la région où l'on vit.}

Un des objectifs poursuivis par la société de géographie est de développer la connaissance des régions de l'Est, et de susciter sur elles des études. Le bulletin est largement ouvert aux études régionales, mais on a beaucoup de mal à trouver des contributions de qualité. Barbier le reconnaît sans peine, à l'assemblée du 28.1.1892: « on a pu souhaiter, longtemps en vain, que la géographie régionale tienne une place convenable dans notre publication ; jusqu'au moment où nous commençâmes celle des travaux de l'instituteur Olry d'Allain (c'est-à-dire en 1883), notre bulletin ne contenait en effet rien ou peu de chose de cette nature ». Mais ce que l'on publie sur la région, le plus souvent en géographie historique, contient une grande part de compilations et peu de recherches originales. On a de la peine à s'écarter du modèle de la monographie. Ce sont d'ailleurs des monographies locales, rédigées par les instituteurs à l'occasion de l'exposition de 1889 , qui paraissent dans le bulletin sous la forme de résumés. On espère sans doute ainsi encourager les écoles primaires à adhérer à la société, en bénéficiant d'un coût d'abonnement allégé. Mais cette forme de vulgarisation, qui fait passer pour de la géographie des catalogues d'informations diverses, n'est en rien propre à faire avancer l'intelligence de cette discipline.

\section{B. Le début d'une vraie réflexion sur la géographie}

41 Elle prend appui à la fois sur la connaissance que l'on peut avoir de la géographie germanique et sur les premiers travaux des grands maîtres parisiens de la géographie universitaire.

\section{Le modèle allemand et autrichien : la conférence de F. de Hellwald}

Barbier se veut un patriote "sans illusion "; il n'entretient pas l'esprit de revanche et croit que les rivalités nationales peuvent être dépassées. La société de géographie de Nancy entretient de bons rapports, non seulement avec les géographes de culture française restés en Alsace, comme Charles Grad (mort en 1890), mais aussi avec la société de géographie de Berlin et avec la revue «Ausland». En 1880, il invite pour une conférence à Nancy F. de Hellwald, qui expose «Comment on étudie la géographie en Allemagne et en Autriche ». Elle a un grand retentissement. Le conférencier montre clairement la voie que doit suivre la géographie pour devenir une science. Elle doit en premier lieu se faire comparative, car la seule manière d'établir des lois générales est de relever qu'un même phénomène peut être commun à différentes parties du globe. Elle doit s'appuyer sur de bonnes cartes, qu'il faut dresser sur des bases mathématiques exactes. Elle doit enfin reposer sur la géographie physique, à laquelle concourent toutes les sciences naturelles, et qui est la base de notre connaissance de la terre.

Un tel langage est sans doute prématuré pour un auditoire que rien n'y a préparé. Barbier ne retient guère de cette conférence que l'infériorité française dans le domaine de la cartographie. Au début de 1890, dans son rapport général sur l'année 1889, il met encore particulièrement l'accent sur ce point. « Pénétré de la nécessité de lutter de pair 
avec la cartographie allemande, et de donner un essor plus grand à la production et à la vulgarisation des cartes géographiques en les rendant meilleures et tout à la fois meilleur marché... », il ambitionne de publier « une revue géographique française de la valeur des Mittheilungen de Gotha, et tous ouvrages semblables de l'Institut fondé par Julius Perthes ». Une commission est constituée pour s'occuper de cette question.

\section{Un disciple isolé de Levasseur : Charles Eugène Génin.}

Charles Eugène Génin, né dans les Vosges en 1838, est professeur au lycée de Nancy. Il a pris une part active à la création de la société, mais a demandé d'être relevé de ses responsabilités au comité en 1882. Ses travaux personnels portent sur l'histoire et la géographie coloniale. Il partage l'opinion générale sur l'utilité de la géographie commerciale, mais il ne la conçoit pas comme une simple vulgarisation de notions élémentaires : il la veut réfléchie, explicative et formatrice pour l'intelligence. Il se réclame ouvertement pour cela de son maitre Émile Levasseur. Dans un compte-rendu d'ouvrage paru en 1880, il rappelle que ce dernier a vivement réagi contre la façon dont on réduisait, avant 1870, la géographie à une simple nomenclature. "Il s'est efforcé, dans des livres clairs, méthodiques, d'une science aussi profonde que précise, de rattacher les faits particuliers à des idées générales pour les faire mieux comprendre ; il a montré la corrélation qui existe entre les productions agricoles, l'industrie et le commerce de chaque contrée ; il a caractérisé chaque région naturelle d'une façon à la fois si exacte et si nette, que les traits essentiels de sa description se gravent facilement dans l'esprit. S'aidant des sciences naturelles, de l'histoire, de l'économie politique et de la statistique, il fait voir la puissance de l'homme sur la nature et réussit, par la variété des aperçus et le piquant des détails, à rendre la géographie utile, pratique, intéressante ». On ne peut plus clairement prendre position: la géographie transmet une science, mais avec les moyens qui sont ceux de l'art pour composer un tableau.

C'est le même Génin qui publie en 1890 à Nancy un ouvrage intitulé «Introduction à l'enseignement de la géographie", préfacé par Levasseur. Bien qu'il en soit encore membre, il n'adresse pas cet ouvrage à la société de géographie. Celle-ci publie tout de même dans son bulletin un compte-rendu qui est extrait du Progrès de l'Est. La définition que Génin donne de la géographie est une profession de foi contre le déterminisme et en faveur de la liberté humaine. "La géographie se résume dans l'action réciproque de l'homme sur la nature et de la nature sur l'homme; Pourvu que le climat ne soit pas malsain ou excessif, et le sol absolument rebelle à toute culture, un peuple, quelle que soit la terre où il habite, est maitre de sa destinée ». En lisant ces lignes, on peut regretter que Génin n'ait pas davantage inspiré la ligne de conduite de la société dans les années 1880 .

\section{L'irruption de la géographie physique sous une forme inattendue à Nancy : l'océanographie}

En 1889, un nouveau professeur de la faculté des sciences de Nancy, Jean Thoulet, expose dans le bulletin ce qu'est l'océanographie, dont il occupe la première chaire. Il souligne qu'en raison de son caractère de rigueur, il faut la classer parmi les sciences physiques plutôt que dans les sciences naturelles. Elle n'est pas la géographie physique, même restreinte au domaine de la mer, mais ajoute-t-il: "toutes les sciences se touchent et se confondent en quelques-uns de leurs points, puisque toutes se proposent 
de connaître la nature qui est une, c'est pourquoi il serait puéril de cantonner chacune d'elles dans un champ rigoureusement limité ». L'année suivante, Thoulet livre au bulletin de la société les carnets de son « Voyage à Terre-Neuve». C'est en réalité le récit d'une longue expédition scientifique sur le navire Clorinde, pendant l'été 1886. Thoulet s'y révèle un observateur remarquable et utilise sa culture scientifique pour expliquer les paysages et les mœurs des habitants; il donne la démonstration vivante de ce que devrait être la géographie du voyageur, qui saisit d'un coup d'œil les faits significatifs et trouve dans les conversations avec les habitants l'interprétation de ceux qui l'intriguent. Il possède ce qui manque à la plupart des géographes de cette époque : le contact prolongé, formateur, avec un terrain nouveau, qu'on découvre et qu'on apprend à aimer peu à peu. Ce scientifique de très haut niveau, associé aux campagnes du prince de Monaco, est géographe dans l'âme.

47 Avec un tel maître, humain, spirituel, pétillant d'intelligence, qui déclare avec humour qu'il a commencé à apprendre l'océanographie au fond des lacs suisses, lieu de ses premières études, la géographie physique ne peut manquer de séduire. Le deuxième jeudi de janvier 1892, dans l'amphithéâtre de sciences naturelles totalement bondé, où se presse une société d'élite et où les femmes sont nombreuses, Thoulet inaugure un cours public de géographie physique. Sa parole est brillante et il donne une éblouissante démonstration de ce que doit être cette discipline. Elle part simplement d'un certain nombre événements qui sont des faits d'observation. Ils ont un caractère dynamique : «ils s'accomplissent autour de nous, à la surface de la terre, à l'instant même où nous sommes, et ils en modifient sans cesse le modelé ». Pour les expliquer et les comprendre, elle fait appel à des mesures exactes, mais aussi à la méthode expérimentale, « à laquelle rien ne résiste quand elle peut être appliquée : elle quitte la montagne, le fleuve, le lac ou l'océan pour entrer dans le laboratoire et, réciproquement, elle passe du laboratoire dans les déserts, sur les falaises, au milieu des déserts ». Mais l'explication de ce que nous voyons n'est complète que si nous acceptons aussi une perspective historique : «Sur le globe, l'œuvre d'hier n'est autre que l'œuvre d'aujourd'hui... La géographie physique, en lisant sur les collines, sur les rivages et jusqu'au fond des abîmes de la mer, y lit aussi l'histoire du passé, et comme ce qui s'accomplit aujourd'hui sous nos yeux se continuera demain..., elle voit en avant aussi bien qu'en arrière ; du présent elle conclut au passé, et du passé à l'avenir ; elle est plus qu'une simple connaissance, elle est exacte, elle est précise, elle énonce des lois, elle est digne de porter le nom de science ».

Pour Auerbach, qui occupe la chaire de géographie de la faculté des Lettres, Thoulet n'est pas un concurrent, mais un appui. Ses étudiants suivent l'enseignement de Thoulet et acquièrent ainsi une excellente base pour leurs études en géographie humaine. Les rapports entre les deux hommes semblent avoir été bons : lorsque parait "Le plateau lorrain" d'Auerbach, Thoulet en fait un bon compte-rendu. Il loue également l'article qu'Auerbach fait paraître sur l'hydrologie du Rhin, d'après des sources allemandes.

\section{Auerbach et la géographie régionale}

Arrivé discrètement à la faculté des Lettres de Nancy, où il occupe la chaire qui avait été celle de Vidal de la Blache et de Marcel Dubois, Auerbach a déjà réfléchi profondément sur l'essence de la géographie, dans sa thèse complémentaire consacrée à Strabon. Affecté d'une assez mauvaise vue, il est un homme de cabinet. Il commence à 
publier en 1890, dans la Revue de Géographie de Drapeyron, une série d'articles intitulée : «La Lorraine, essai de chorographie ». Il se place d'emblée sur le terrain de la géographie "scientifique », comme le souligne dans son compte rendu du Bulletin de la société de géographie de l'Est Charles Millot: «c'est l'occasion de rappeler que la géographie scientifique émane de la Faculté de Nancy, par M. Vidal de la Blache d'abord, puis par ses élèves, Dubois, Auerbach, Poirel. Il importe de mettre en relief ce fait trop peu connu de nos concitoyens ». Lors d'une livraison suivante, en 1891, Charles Millot définit avec beaucoup de finesse la méthode d'Auerbach, qui consiste en une suite de synthèses partielles, dans lesquelles toutes les relations de causalité sont abondamment soulignées. "L'auteur se garde bien de diviser en chapitres les choses qu'on ne peut isoler, puisqu'elles s'enchaînent et s'entremêlent si bien qu'elles réagissent constamment les unes sur les autres, au point d'être réciproquement effets et causes. Il a préféré faire du tout une description synthétique et raisonnée, qui semble une peinture à la fois panoramique et détaillée ».

Lorsqu'en 1893 les articles de la " Revue de Géographie » sont réunis en un livre, dont Barbier exécute les croquis, le compte-rendu est cette fois l'œuvre de Jean Thoulet, qui en quelques lignes très incisives, met parfaitement l'accent sur les qualités propres à Auerbach : son "aptitude à des connaissances fort diverses ", qui traduit sa grande curiosité intellectuelle, l'habileté avec laquelle il traite les différentes spécialités dont il se sert pour élucider son sujet et particulièrement le talent avec lequel il manie les statistiques. À ce propos, Thoulet ne se prive pas d'envoyer un petit coup de griffe à la géographie humaine : elle doit s'appuyer sur des statistiques établies à l'intérieur de frontières politiques changeantes et qui ne correspondent pas à des limites naturelles ; il faut les adapter pour les appliquer aux espaces qu'étudie la géographie. En lisant tous ces éloges, on se dit qu'Auerbach est un virtuose, difficilement imitable: cette géographie n'est-elle pas réservée à une élite très cultivée, et n'est-elle pas aux antipodes de la vulgarisation dont rêvaient Barbier et ses amis?

\section{Un âge d'or de la géographie à Nancy ? (1900-1914)}

51 Dans les premières années du XXe siècle, la fièvre coloniale est quelque peu retombée. Le milieu dans lequel avaient grandi Hubert Lyautey et Charles de Foucauld a évolué. Nancy ne s'enflamme plus comme dans les années 1880 et 1890 pour les exploits de hardis pionniers dont on attend avec impatience toutes les correspondances. L'aventure scientifique a pris le pas sur l'aventure coloniale pure, le commandant Charcot (invité par la société en 1906) remplace comme héros modèle Savorgnan de Brazza. Les colonies sont maintenant considérées surtout comme un prolongement économique de la métropole. Elles ne sont plus, pour les jeunes Lorrains, ce que l'on pourrait appeler, d'un mot anachronique mais juste, une "nouvelle frontière ». La population se préoccupe maintenant davantage de la frontière toute proche, d'où viennent les menaces. Elle prend conscience du fait que la Lorraine possède d'énormes possibilités de développement économique, grâce à son riche gisement de fer, mais en même temps des risques que cette prospérité, propre à éveiller les jalousies du voisin, peut lui faire courir. La géographie joue son rôle dans ce recentrage qui n'est pas un simple repli sur soi.

52 En 1909, les géographes nancéiens reçoivent pour la deuxième fois la charge d'organiser le congrès annuel des sociétés de géographie françaises. Cette réunion 
coïncide fort heureusement avec la grande Exposition internationale de l'Est de la France, qui a marqué un véritable sommet dans le rayonnement industriel et artistique de Nancy au début du XXe siècle. Cela montre bien la volonté des géographes d'apporter leur contribution au succès de cette grande manifestation et à son rayonnement. Sous la houlette d'Auerbach, ils sont rangés en ordre de bataille, pour bien faire apparaître toutes les facettes de leurs activités. Thoulet fait visiter son laboratoire et replace l'océanographie dans l'histoire de la terre. Imbeaux explique la distribution d'eau potable et la constitution du système des égouts à Nancy. Joly montre le relief de la Lorraine en se servant d'un plan géologique en relief. Villain, ingénieur en chef des mines, présente le stand des mines de fer à l'Exposition internationale. Auerbach, quant à lui, dépeint la Lorraine comme un «Transvaal français » et montre de façon très juste le lien qui existe entre l'université et le développement économique. Sans elle, il n'y aurait pas eu tous ces ingénieurs, ces chimistes, ces mécaniciens. Mais, dit-il, à leur tour leurs travaux ont permis de faire beaucoup progresser la connaissance de la région. « Nous devons les investigations géographiques dont le territoire lorrain a été l'objet à l'université moderne. C'est elle qui nous a amené tant de richesses ». Louis Lafitte, secrétaire de la Chambre de commerce de Nancy et géographe de qualité, a été la cheville ouvrière de l'Exposition; il porte les projets de développement des voies de communication dans la France de l'Est. La géographie que l'on présente en 1909 est donc une géographie active, autant qu'appliquée, qui continue à canaliser toutes sortes de courants. Bien qu'elle ait corrigé son laxisme primitif, elle garde néanmoins un grand pouvoir fédérateur.

\section{Conclusion}

53 S'il a existé vers 1900 une "école " artistique de Nancy, il n'y a jamais eu d' "école géographique » dans cette ville avant 1914 et il n'y a sans doute pas lieu de le regretter. Ni Auerbach, ni Thoulet, ni Millot, ni aucun autre n'ont voulu imposer leur façon de voir. Chacun travaille à sa manière, du mieux qu'il peut, mais sans se couper ni de ses collègues, ni du mouvement géographique international, dont on suit l'évolution à travers les grands congrès. Personne ne songerait à prendre toute son inspiration à Paris, même si l'on connaît et respecte les maîtres parisiens. Ce sont d'ailleurs les mêmes qui réclament à la capitale un peu plus de "décentralisation ». Le champ de la géographie est largement ouvert, il s'étend à de nombreux domaines et à toutes les parties du monde. Chacun peut travailler librement, sans risquer d'être gêné par des voisins, qui deviendraient des concurrents. Au contraire, toutes les collaborations sont acceptées.

S'il n'y a pas une « école » géographique nancéienne, il existe pourtant un « modèle » nancéien : celui d'une géographie ouverte, multiple, conviviale, à l'écoute de l'opinion publique, qui lui apporte en retour son soutien. À un siècle de distance, en évoquant ce que ce passé a eu de richesse, on mesure ce que les géographes français ont perdu en audience, à force de s'enfermer dans leurs chapelles et de cultiver leurs étroites spécialités. 


\section{BIBLIOGRAPHIE}

La bibliographie de ce travail consiste essentiellement dans le dépouillement exhaustif du Bulletin de la Société de géographie de l'Est, publié à Nancy de 1879 à 1914. La société a continué d'organiser des conférences à Nancy après 1918, mais a cessé de publier son bulletin après la Première Guerre mondiale.

\section{RÉSUMÉS}

Nancy a participé, à partir de 1879, au mouvement qui a donné naissance à de nombreuses sociétés de géographie dans les principales villes françaises de province. Grâce à l'activité inlassable de son fondateur, J.V.Barbier, la société a connu un très vif succès dans les années 1880 et fondé deux sociétés sœurs à Bar-le-Duc et Épinal. Le nombre de ses membres a diminué fortement de 1890 à 1914, en dépit d'un sursaut de 1900 à 1905. La qualité du bulletin publié par la société a été très inégale. Dans les premières années, sous la direction de Barbier, il accueille des contributions très disparates, parfois exagérément longues et de valeur discutable, mais qui répondent au goût du public. Après la mort de Barbier, les universitaires prennent en main la rédaction et imposent des articles plus courts, mieux classés, porteurs d'un contenu scientifique plus réel. Il est intéressant de voir comment à Nancy s'est constitué un noyau de savants, dont les préoccupations s'ouvraient vers toutes les disciplines composant la géographie ; la géographie physique était la mieux représentée, grâce à l'excellente collaboration établie entre les facultés des Lettres et des Sciences; mais la géographie humaine tenait également sa place, grâce à B. Auerbach et avec le renfort d'historiens, de voyageurs et d'hommes d'affaires. Dans ce creuset nancéien, original et dynamique, émerge peu à peu une certaine conception de la géographie. Elle s'inspire dans ses grandes lignes de l'école de géographie scientifique née à Paris autour des «Annales de Géographie ». Mais les géographes nancéiens conservent tout de même par rapport à Paris une certaine autonomie, grâce à la qualité de leur équipe et grâce aux relations directes établies avec des savants allemands et anglo-américains. On ne peut pas parler d'une «école » de Nancy en matière de géographie, mais d'un modèle ouvert, convivial et dynamique, d'une géographie vivante parce qu'associée à la vitalité régionale de la Lorraine à cette époque.

Nancy has contributed, from 1879 , to the movement which founded geographical societies in the main french provincial towns. Owing to the indefatigable work of its founder, J.V. Barbier, the society was very successful in the 1880 s and aided the birth of its two sistersocieties, at Bar-leDuc and Epinal. The number of members fell heavily between 1890 and 1914, in spite of a burst of energy from 1900 to 1905 . The quality of papers published by the society was very uneven. In the early years, under the direction of Barbier, the "Bulletin » received a very ill-assorted set of contributions, sometimes too long or of doubtful value, but corresponding to public taste. After Barbier's death, the university professors took the writing in hand and insisted on better, shorter articles, with a more scientific content. It is interesting to see how at Nancy was constituted, then here developed a hard core of scientists, who were open-minded about all aspects of geographical sciences; physical geography was the better represented, owing to the excellent partnership between the two faculties of Letters and Sciences; but human geography also played its part, represented by B.Auerbach, with the assistance of historians, travellers and businessmen. In that original and dynamic nancian crucible emerged, little by little, a true conception of Geography. Its inspiration is in the grand line of the school of scientific Geography born in Paris around the «Annales de géographie». But the nancian geographers retain yet a relative autonomy from 
Paris, because of the rich diversity of their team and their direct relations with German and Anglo-american scientists. One cannot speak of a nancian « school » as regards Geography, but of an open-minded, convivial and dynamic linely Geography because of its association with the regional vitality of Lorraine at that time.

Nancy hat nach $1879 \mathrm{zu}$ der Entwicklung beigetragen, die zahlreiche geographische Gesellschaften in den französischen Provinzhauptstädten begründet hat. Dank der unermüdlichen Arbeit ihres Gründers, J.V.Barbier, hat die Gesellschaft von Nancy in den Jahren um 1880 einen grossen Erfolg erzielt und zwei Schwestergesellschaften in Bar-le-Duc und Epinal gegründet. Die Zahl der Mitglieder ist zwischen 1890 und 1914 stark gefallen, trotz einem kurzen Wiederaufstieg in den Jahren 1900 bis 1905. Die Qualität der veröffentlichen Beiträge war sehr ungleich. Am Anfang enthielt die unter der Leitung von Barbier verlegte Zeitschrift sehr verschiedene Beiträge, manchmal zu lang und von fraglichem Wert, aber zum allgemeinen Geschmack passend. Nachdem Barbier gestorben war, forderten die Universitätsprofessoren, die den Redaktionsausschuss bildeten, kürzere Beiträge mit einem besseren wissenschaftlichen Gehalt. Es ist interessant zu sehen, wie sich in Nancy eine Gruppe von Gelehrten bildete, die zugänglich für alle Fächer der geographischen Wissenschaft war. Die physische Geographie war am besten repräsentiert, dank der guten Zusammenarbeit zwischen den beiden Fakultäten der Literatur und der Naturwissenschaften. Die Anthropogeographie spielte aber auch eine gewisse Rolle, dank B.Auerbachs, und mit Hilfe der Historiker Reisenden und Geschäftsleute. In diesem originellen und tatkräftigen «Schmelztiegel » von Nancy kommt allmählich eine bestimmte Konzeption der Geographie zum Vorschein. Sie wird in grossen Zügen von der neuen wissenschaftlichen Schule der Geographie beeinflusst, die sich in Paris in den «Annales de géographie » entwickelte. Aber die lothringischen Geographen bewahrten doch eine gewisse Selbstständigkeit, dank der Qualität ihrer Mitglieder und dank ihrer unmittelbaren Beziehungen zu deutschen und angloamerikanischen Gelehrten. Man kann nicht von einer echten " geographischen Schule » in Nancy sprechen, sondern von eine offenen, gastfreundlichen und dynamischen Atmosphäre. Diese Erdkunde war mit der geistigen und wirtschaftlichen Lebenskraft der Region Lothringen eng verbunden.

\section{INDEX}

Schlüsselwörter : Epistemologie (Wissenschaftstheorie) der Erdkunde, Geschichte der Erdkunde, Lothringen, Nancy, regionale erdkundliche Gesellschaften

Keywords : epistemology of geography., history of geography, Lorraine, Nancy, regional geographical societies

Mots-clés : épistémologie de la géographie, histoire de la géographie, Lorraine, Nancy, sociétés régionales de géographie

\section{AUTEUR}

\section{JEAN-CLAUDE BONNEFONT}

Professeur à l’Université de Nancy 2 - Institut de géographie - BP 339754015 Nancy CEDEX 